\title{
Accelerated fitted operator finite difference method for singularly perturbed delay differential equations with non-local boundary condition
}

\author{
Habtamu Garoma Debela and Gemechis File Duressa*
}

* Correspondence: gammeef@ yahoo.com

Department of Mathematics, Jimma University, P. O. Box 378, Jimma, Ethiopia

\begin{abstract}
In this paper, accelerated fitted finite difference method for solving singularly perturbed delay differential equation with non-local boundary condition is considered. To treat the non-local boundary condition, Simpson's rule is applied. The stability and parameter uniform convergence for the proposed method are proved. To validate the applicability of the scheme, two model problems are considered for numerical experimentation and solved for different values of the perturbation parameter $\varepsilon$ and mesh size $h$. The numerical results are tabulated in terms of maximum absolute errors and rate of convergence, and it is observed that the present method is more accurate and $\varepsilon$-uniformly convergent for $h \geq \varepsilon$ where the classical numerical methods fails to give good result, and it also improves the results of the methods existing in the literature.
\end{abstract}

Keywords: Singularly perturbed problems, Delay differential equation, Fitted finite difference, Non-local boundary condition

Mathematics Subject Classification: 65L11, 65L12, 65L20

\section{Introduction}

A differential equation is said to be singularly perturbed delay differential equation, if it includes at least one delay term, involving unknown functions occurring with different arguments, and also, the highest derivative term is multiplied by a small parameter. Such type of delay, differential equations play a very important role in the mathematical models of science and engineering, such as, the human pupil light reflex with mixed delay type [1], variational problems in control theory with small state problem [2], models of HIV infection [3], and signal transition [4]. Any system involving a feedback control almost involves time delay. The delay occurs because a finite time is required to sense the information and then react to it. Finding the solution of singularly perturbed delay differential equations, whose application mentioned above, is a challenging problem. In response to these, in recent years, there has been a growing interest in numerical methods on singularly perturbed delay differential equations. The authors of [5-7] have developed various

(c) The Author(s). 2020 Open Access This article is licensed under a Creative Commons Attribution 4.0 International License, which permits use, sharing, adaptation, distribution and reproduction in any medium or format, as long as you give appropriate credit to the original author(s) and the source, provide a link to the Creative Commons licence, and indicate if changes were made. The images or other third party material in this article are included in the article's Creative Commons licence, unless indicated otherwise in a credit line to the material. If material is not included in the article's Creative Commons licence and your intended use is not permitted by statutory regulation or exceeds the permitted use, you will need to obtain permission directly from the copyright holder. To view a copy of this licence, visit http://creativecommons.org/licenses/by/4.0/. 
numerical schemes on uniform meshes for singularly perturbed first and second order differential equations with integral boundary conditions. The authors of [8-10] have proved that the problem of differential equations with integral boundary conditions is well posed. The authors of [11] proposed a first order uniform convergent fitted finite difference scheme for singularly perturbed boundary value problem for a linear second order delay differential equation with large delay in reaction term.

The standard numerical methods used for solving singularly perturbed differential equation are sometime ill posed and fail to give analytical solution when the perturbation parameter $\varepsilon$ is small.

Therefore, it is necessary to develop suitable numerical methods which are uniformly convergent to solve this type of differential equations. In [12-15], finite difference and finite element methods are proposed to solve this kind of equations with large and small shifts.

As far as the researchers' knowledge is concerned, numerical solution of singularly perturbed boundary value problem containing integral boundary condition via accelerated exponential fitted operator method is first being considered. The basic essence of accelerated fitted operator finite difference method is fitting an operator into a finite difference scheme, determining the value for the operator, formulating the intended scheme, and then applying Richardson extrapolation that can be explained as whenever the leading term in the error for an approximation formula is known; we can combine two approximations obtained from the formula using different values of the mesh sizes $h$ and $0.5 h$ to obtain a higher order approximation, and the technique is known as Richardson extrapolation. This procedure is a convergence acceleration technique which consists of a linear combination of two computed approximations of a solution (applied on two nested meshes). The linear combination turns out to be a better approximation. Therefore, the main objective of this study is to develop $\varepsilon$-uniformly convergent and more accurate numerical method for solving singularly perturbed delay differential equations with non-local boundary condition. Hence, in the present paper, motivated by the works of [16], we developed a fitted operator finite difference scheme on uniform mesh for the numerical solution for second order singularly perturbed convection-diffusion equations with negative shift and non-local boundary condition.

The present paper is organized as follows. Statement of the problem is given in the "Statement of the problem" section. In the "Properties of continuous solution" section, properties of continuous solution are presented. The "Formulation of the numerical scheme" section describes formulation of the numerical scheme. Convergence analysis for approximate solution is given in the "Convergence analysis" section. Numerical results are given in the "Numerical examples and results" section. Discussion and conclusion is given in the "Discussion and conclusion" section.

Throughout our analysis, $C$ is a generic positive constant that is independent of the parameter $\varepsilon$ and number of mesh points $2 N$. We assume that $\bar{\Omega}=[0,2], \Omega=(0,2)$, $\Omega_{1}=(0,1), \Omega_{2}=(1,2)$. Further, $\Omega^{*}=\Omega_{1} \cup \Omega_{2}, \bar{\Omega}^{2 N}$ is denoted by $\{0,1,2, \ldots, 2 N\}$, $\bar{\Omega}_{1}^{2 N}$ is denoted by $\{1,2, \ldots, N-1\}, \Omega_{2}^{2 N}$ is denoted by $\{N+1, N+2, \ldots, 2 N-1\}$.

\section{Statement of the problem}

Consider the following singularly perturbed problem: 


$$
\begin{aligned}
& L y(x)=-\varepsilon y^{\prime \prime}(x)+a(x) y^{\prime}(x)+b(x) y(x)+c(x) y(x-1)=f(x), x \in \Omega=(0,2), \\
& y(x)=\phi(x), \quad x \in[-1,0] \\
& K y(2)=y(2)-\varepsilon \int_{0}^{2} g(x) y(x) d x=l
\end{aligned}
$$

where $\phi(x)$ is sufficiently smooth on $[-1,0]$. For all $x \in \Omega$, it is assumed that the sufficient smooth functions $a(x), b(x)$ and $c(x)$ satisfy $a(x)>\alpha_{1}>\alpha>0, b(x) \geq \beta \geq 0, c(x) \leq \gamma \leq$ 0 , and $\alpha+\beta+\gamma>0$. Furthermore, $g(x)$ is non-negative and monotonic with $\int_{0}^{2} g(x) d x$ $<1$. The above assumptions ensure that $y \in X=C^{0}(\Omega) \cap C^{1}(\Omega) \cap C^{2}\left(\Omega_{1} \cup \Omega_{2}\right)$ [16].

Eqs. (1)-(3) is equivalent to

$$
L y(x)=F(x)
$$

where

$$
\begin{aligned}
& L y(x)= \begin{cases}L_{1} y(x)=-\varepsilon y^{\prime \prime}(x)+a(x) y^{\prime}(x)+b(x) y(x), & x \in \Omega_{1}=(0,1) \\
L_{2} y(x)=-\varepsilon y^{\prime \prime}(x)+a(x) y^{\prime}(x)+b(x) y(x)+c(x) y(x-1), & x \in \Omega_{2}=(1,2)\end{cases} \\
& F(x)= \begin{cases}f(x)-c(x) \phi(x-1), & x \in \Omega_{1} \\
f(x), & x \in \Omega_{2}\end{cases}
\end{aligned}
$$

with boundary conditions

$$
\left.\begin{array}{l}
y(x)=\phi(x), x \in[-1,0], \\
y\left(1^{-}\right)=y\left(1^{+}\right), y^{\prime}\left(1^{-}\right)=y^{\prime}\left(1^{+}\right), \\
K y(2)=y(2)-\varepsilon \int_{0}^{2} g(x) y(x) d x=l,
\end{array}\right\}
$$

\section{Properties of continuous solution}

Lemma 1: (Maximum Principle) Let $\psi(x)$ be any function in $X$ such that $\psi(0) \geq 0$, $K \psi(2) \geq 0, L_{1} \psi(x) \geq 0, \forall x \in \Omega_{1}, L_{2} \psi(x) \geq 0, \forall x \in \Omega_{2}$ and $\left[\psi^{\prime}\right](1) \leq 0$ then $\psi(x) \geq 0, \forall x \in \bar{\Omega}$.

Proof: Define the test function

$$
s(x)= \begin{cases}\frac{1}{8}+\frac{x}{2}, & x \in[0,1] \\ \frac{3}{8}+\frac{x}{4}, & x \in[1,2]\end{cases}
$$

Note that $s(x)>0, \forall x \in \bar{\Omega}, L s(x)>0, \forall x \in \Omega_{1} \cup \Omega_{2}, s(0)>0, K s(2)>0$, and $\left[s^{\prime}\right](1)<0$.

Let $\mu=\max \left\{\frac{-\psi(x)}{s(x)}: x \in \bar{\Omega}\right\}$. Then, there exists $x_{0} \in \bar{\Omega}$ such that $\psi\left(x_{0}\right)+\mu s\left(x_{0}\right)=0$ and $\psi$ $(x)+\mu s(x) \geq 0, \forall x \in \bar{\Omega}$. Therefore, the function $(\psi+\mu s)$ attains its minimum at $x=x_{0}$. Suppose the theorem does not hold true, then $\mu>0$.

Case (i): $x_{0}=0 ; 0<(\psi+\mu s)(0)=\psi(0)+\mu s(0)=0$, it is a contradiction. 
Case (ii): $\quad x_{0} \in \Omega_{1} 0<L(\psi+\mu s)\left(x_{0}\right)=-\varepsilon(\psi+\mu s)^{\prime \prime}\left(x_{0}\right)+a\left(x_{0}\right)(\psi+\mu s)^{\prime}\left(x_{0}\right)+b\left(x_{0}\right)(\psi+$ $\mu s)\left(x_{0}\right) \leq 0$, it is a contradiction.

Case (iii): $x_{0}=1 ; 0 \leq\left[(\psi+\mu s)^{\prime}\right](1)=\left[\psi^{\prime}\right](1)+\mu\left[s^{\prime}\right](1)<0$, it is a contradiction.

Case (iv): $x_{0} \in \Omega_{2}$

$$
\begin{aligned}
0<L(\psi+\mu s)\left(x_{0}\right) & =-\varepsilon(\psi+\mu s)^{\prime \prime}\left(x_{0}\right)+a\left(x_{0}\right)(\psi+\mu s)^{\prime}\left(x_{0}\right)+b\left(x_{0}\right)(\psi+\mu s)\left(x_{0}\right) \\
& +c\left(x_{0}\right)(\psi+\mu s)\left(x_{0}-1\right) \leq 0, \text { it is a contradiction. }
\end{aligned}
$$

Case (v): $\quad x_{0}=2 ; \quad 0 \leq K(\psi+\mu s)(2)=(\psi+\mu s)(2)-\varepsilon \int_{0}^{2} g(x)(\psi+\mu s)(x) d x \leq 0$,

it is a contradiction.

Hence, the proof of the theorem.

Lemma 2: (Stability Result) The solution $y(x)$ for the problems (1)-(3) satisfies the bound

$$
|y(x)| \leq C \max \left\{|y(0)|,|K y(2)|, \sup _{x \in \Omega^{*}}|L y(x)|\right\}, x \in \bar{\Omega}
$$

Proof: This theorem can be proved by using Lemma 1, and the barrier functions $\theta^{ \pm}(x)=C M s(x) \pm y(x), x \in \bar{\Omega}$, where $M=\max \left\{|y(0)|,|K y(2)|, \sup _{x \in \Omega^{*}}|L y(x)|\right\}$ and $s(x)$ are the test function as in Lemma 1.

Lemma 3: Let $y(x)$ be the solution for (1)-(3). Then we have the following bounds:

$$
\left\|y^{(k)}(x)\right\|_{\Omega^{*}} \leq C \varepsilon^{-k}, \text { for } k=1,2,3 .
$$

Proof: For the proof, refer to [16].

Lemma 4: The bound for derivative of the solution $y(x)$ of the problems (1)-(3) when $x \in \Omega_{1}=(0,1)$ is given by:

$$
\left|y^{(k)}(x)\right| \leq C\left(1+\varepsilon^{-k} \exp \left(\frac{-\alpha\left(1-x_{j}\right)}{\varepsilon}\right)\right), k=0 \leq k \leq 4, \quad j=1,2, \ldots, N-1 .
$$

Proof: For the proof, refer to [17].

\section{Formulation of the numerical scheme}

For small values of $\varepsilon$, the boundary value problem, (1)-(3) exhibit strong boundary layer at $x=2$ and interior layer at $x=1$ (see [16]).

The linear ordinary differential Eq. (1) cannot, in general, be solved analytically because of the dependence of $a(x), b(x)$, and $c(x)$ on the spatial coordinate $x$. We divide the interval $[0,2]$ into $2 N$ equal parts with constant mesh length $h$. Let $0=x_{0}, x_{2}, \ldots$, $x_{N}=1, x_{N+1}, x_{N+2}, \ldots, x_{2 N}=2$ be the mesh points. Then, we have $x_{i}=i h, i=0,1,2$, $\ldots 2 N$. If we consider, the interval $x \in(0,1)$ and the coefficients of $(1)$ are evaluated at the midpoint of each interval; then, we will obtain the differential equation:

$$
\left\{\begin{array}{l}
-\varepsilon y^{\prime \prime}(x)+a(x) y^{\prime}(x)+b(x) y(x)=f(x)-c(x) \phi(x-1), x \in \Omega_{1}=(0,1) \\
y_{0}=y(0)=\phi(0)
\end{array}\right.
$$

Now, the domain $[0,1]$ is discretized into $N$ equal number of subintervals, each of length $h$. Let $0=x_{0}<x_{1}<\ldots<x_{N}=1$ be the points such that $x_{i}=i h, i=0,1,2, \ldots, N$. For 
the discretization, we apply a exponentially fitted operator finite difference method (FOFDM).

From (9), we have

$$
-\varepsilon y^{\prime \prime}(x)+a(x) y^{\prime}(x)+b(x) y(x)=F(x), \quad x \in \Omega_{1}=(0,1)
$$

where $F(x)=f(x)-c(x) \phi(x-1)$.

To find the numerical solution of (10), we use the theory applied in asymptotic method for solving singularly perturbed BVPs. In the considered case, the boundary layer is in the right side of the domain, i.e., near $x=1$. From the theory of singular perturbations given by O'Malley [18] and using Taylor's series expansion for $a(x)$ about $x=1$ and restriction to their first terms, we get the asymptotic solution as follows:

$$
y(x)=y_{0}(x)+\left(\theta-y_{0}(1)\right) \exp \left(-\frac{a(1)(1-x)}{\varepsilon}\right),
$$

where $y_{0}(x)$ is the solution of the reduced problem (obtained by setting $\varepsilon=0$ ) of (10) which is given by:

$$
a(x) y^{\prime}(x)+b(x) y(x)=F(x) \text { with } y_{0}(0)=\phi(0) \text {. }
$$

Considering $h$ is small enough, the discretized form for (11) becomes

$$
y(i h)=y_{0}(i h)+\left(\theta-y_{0}(1)\right) \exp \left(-\frac{a(1)(1-i h)}{\varepsilon}\right),
$$

which is simplified to

$$
y(i h)=y_{0}(i h)+\left(\theta-y_{0}(1)\right) \exp \left(-a(1)\left(\frac{1}{\varepsilon}-i \rho\right)\right),
$$

where $\rho=\frac{h}{\varepsilon}, h=\frac{1}{N}$.

To handle the effect of the perturbation parameter, artificial viscosity (an exponentially fitting factor $\sigma(\rho)$ ) is multiplied on the term containing the perturbation parameter as follows:

$$
-\varepsilon \sigma(\rho) y^{\prime \prime}(x)+a(x) y^{\prime}(x)+b(x) y(x)=F(x),
$$

with boundary conditions $y_{0}(0)=\phi(0)$ and $y(N)=\theta$, where $y(N)$ is evaluated by Runge-Kutta method from the reduced solution of (12).

Next, we consider the difference approximation of (9) on a uniform grid $\bar{\Omega}^{N}$ $=\left\{x_{i}\right\}_{i=0}^{N}$ and denote $h=x_{i+1}-x_{i}$.

For any mesh function $z_{i}$, define the following difference operators:

$$
\begin{aligned}
D^{+} z_{i} & =\frac{z_{i+1}-z_{i}}{h}, D^{-} z_{i}=\frac{z_{i}-z_{i-1}}{h}, D^{0} z_{i}=\frac{z_{i+1}-z_{i-1}}{2 h}, D^{+} D^{-} z_{i} \\
& =\frac{z_{i+1}-2 z_{i}+z_{i-1}}{h^{2}}
\end{aligned}
$$

by applying the central finite difference scheme on (14) takes the form:

$$
-\varepsilon \sigma(\rho)\left(D^{+} D^{-} y\left(x_{i}\right)\right)+a\left(x_{i}\right)\left(D^{0} y\left(x_{i}\right)\right)+b\left(x_{i}\right) y\left(x_{i}\right)=F\left(x_{i}\right),
$$

with the boundary conditions $y_{0}(0)=\phi(0)$ and $y(N)=\theta$.

Using operator, (10) is rewritten as follows:

$$
L^{h} y_{i}=F_{i}
$$

with the boundary conditions $y_{0}=\phi(0)$ and $y_{N}=\theta$. 
where

$$
L^{h} y_{i}=-\varepsilon \sigma(\rho)\left(\frac{y_{i+1}-2 y_{i}+y_{i-1}}{h^{2}}\right)+a\left(x_{i}\right)\left(\frac{y_{i+1}-y_{i-1}}{2 h}\right)+b\left(x_{i}\right) y_{i}=F_{i},
$$

multiplying (18) by $h$ and considering $h$ is small and truncating the term $h\left(F_{i}-\right.$, result

$$
\frac{\sigma(\rho)}{\rho}\left(y_{i-1}-2 y_{i}+y_{i+1}\right)+\frac{a\left(x_{i}\right)}{2}\left(y_{i-1}-y_{i+1}\right)=0 .
$$

Now, by using Taylor's series for $y_{i-1}$ and $y_{i+1}$ up to first term and substituting the results in (19) into (16) and simplifying, the exponential fitting factor is obtained as follows:

$$
\sigma(\rho)=\frac{\rho a(1)}{2} \operatorname{coth}\left(\frac{\rho a(1)}{2}\right) .
$$

Assume that $\bar{\Omega}^{2 N}$ denotes partition of [0,2] into $2 N$ subintervals such that $0=x_{0}<$ $x_{1}<\ldots<x_{N}=1$ and $1<x_{N+1}<x_{N+2}<\ldots<x_{2 N}=2$ with $x_{i}=i h, h=\frac{2}{2 N}=\frac{1}{N}, i=0,1,2, \ldots$ , $2 N$.

Case 1: Consider (4) on the domain $\Omega_{1}=(0,1)$ which is given by:

$$
-\varepsilon y^{\prime \prime}(x)+a(x) y^{\prime}(x)+b(x) y(x)=f(x)-c(x) \phi(x-1)
$$

Hence, the required finite difference scheme becomes

$$
\begin{aligned}
& \left(\frac{-\varepsilon \sigma(\rho)}{h^{2}}-\frac{a\left(x_{i}\right)}{2 h}\right) y_{i-1}+\left(\frac{2 \varepsilon \sigma(\rho)}{h^{2}}+b\left(x_{i}\right)\right) y_{i}+\left(\frac{-\varepsilon \sigma(\rho)}{h^{2}}+\frac{a\left(x_{i}\right)}{2 h}\right) y_{i+1} \\
& \quad=f_{i}-c_{i} \phi\left(x_{i}-N\right)
\end{aligned}
$$

for $i=0,1,2, \ldots, N$.

The numerical scheme in (22) can be written in three-term recurrence relation as follows:

$$
E_{i} y_{i-1}+F_{i} y_{i}+G_{i} y_{i+1}=H_{i}, \quad i=1,2, \ldots, N,
$$

where $E_{i}=\frac{-\varepsilon \sigma}{h^{2}}-\frac{a_{i}}{2 h}, \quad F_{i}=\frac{2 \varepsilon \sigma}{h^{2}}+b_{i}, \quad G_{i}=\frac{-\varepsilon \sigma}{h^{2}}+\frac{a_{i}}{2 h}, H_{i}=f_{i}-c_{i} \phi\left(x_{i}-N\right)$.

Case 2: Consider (4) on the domain $\Omega_{2}=(1,2)$, for right layer in the domain $\Omega_{2}$ using exponentially fitted finite difference method, which is given by:

$$
-\varepsilon \sigma(\rho)\left(\frac{y_{i+1}-2 y_{i}+y_{i-1}}{h^{2}}\right)+a_{i}\left(\frac{y_{i+1}-y_{i-1}}{2 h}\right)+b_{i} y_{i}+c_{i} y\left(x_{i}-1\right)+\tau_{1}=f_{i} .
$$

Similarly, this equation can be written as follows:

$$
c_{i} y_{j}+E_{i} y_{i-1}+F_{i} y_{i}+G_{i} y_{i+1}=H_{i}, \quad i=N+1, N+2, \ldots, 2 N-1,
$$

where $y_{j}=y\left(x_{i}-1\right), \quad j=1,2, \ldots, N$

$$
E_{i}=\frac{-\varepsilon \sigma}{h^{2}}-\frac{a_{i}}{2 h}, \quad F_{i}=\frac{2 \varepsilon \sigma}{h^{2}}+b_{i}, \quad G_{i}=\frac{-\varepsilon \sigma}{h^{2}}+\frac{a_{i}}{2 h}, \quad H_{i}=f_{i} .
$$

Case 3: For $i=2 N$, the composite Simpson's rule approximates the integral of $g(x) y(x)$ by: 


$$
\int_{0}^{2} g(x) y(x) d x=\frac{h}{3}\left(g(0) y(0)+g(2) y(2)+2 \sum_{i=1}^{2 N-1} g\left(x_{2 i}\right) y\left(x_{2 i}\right)+4 \sum_{i=1}^{2 N} g\left(x_{2 i-1}\right) y\left(x_{2 i-1}\right)\right) .
$$

Substituting (25) into (3) gives:

$$
y(2)-\frac{\varepsilon h}{3}\left(g(0) y(0)+g(2) y(2)+2 \sum_{i=1}^{2 N-1} g\left(x_{2 i}\right) y\left(x_{2 i}\right)+4 \sum_{i=1}^{2 N} g\left(x_{2 i-1}\right) y\left(x_{2 i-1}\right)\right)=L
$$

Since $y(0)=\phi(0)$, from (2), this equation can be re-written as follows:

$$
-\frac{4 \varepsilon h}{3} \sum_{i=1}^{2 N} g\left(x_{2 i-1}\right) y\left(x_{2 i-1}\right)-\frac{2 \varepsilon h}{3} \sum_{i=1}^{2 N-1} g\left(x_{2 i}\right) y\left(x_{2 i}\right)+\left(1-\frac{\varepsilon h}{3} g(2)\right) y(2)=L+\frac{\varepsilon h}{3} g(0) y(0) .
$$

Therefore, on the whole domain $\bar{\Omega}=[0,2]$, the basic schemes to solve (1)-(3) are the schemes given in (23), (24), and (26) together with the local truncation error of $\tau_{1}$.

\section{Convergence analysis}

The discrete scheme corresponding to the original problem (1)-(3) is as follows:

$$
\begin{aligned}
& \text { For } i=1,2, \ldots, N-1, \quad L_{1}^{N} Y_{i}=f_{i}-b_{i} \phi_{i-N}, \\
& \text { For } i=N+1, \ldots, 2 N-1, \quad L_{2}^{N} Y_{i}=f_{i},
\end{aligned}
$$

subject to the boundary conditions is as follows:

$$
\begin{aligned}
& Y_{i}=\phi_{i}, i=-N,-N+1, \ldots, 0, \\
& K^{N} Y_{2 N}=Y_{2 N^{-}} \sum_{i=1}^{2 N} \frac{g_{i-1} Y_{i-1}+4 g_{i} Y_{i}+g_{i+1} Y_{i+1}}{3} h_{i},
\end{aligned}
$$

and

$$
D^{-} Y_{N}=D^{+} Y_{N}
$$

where

$$
\begin{aligned}
& L_{1}^{N} Y_{i}=-\varepsilon \delta^{2} Y\left(x_{i}\right)+a\left(x_{i}\right) D^{0} Y\left(x_{i}\right)+b\left(x_{i}\right) Y\left(x_{i}\right) \\
& L_{2}^{N} Y_{i}=-\varepsilon \delta^{2} Y\left(x_{i}\right)+a\left(x_{i}\right) D^{0} Y\left(x_{i}\right)+b\left(x_{i}\right) Y\left(x_{i}\right)+c\left(x_{i}\right) Y\left(x_{i-N}\right)
\end{aligned} .
$$

Lemma 5: (Discrete Maximum Principle) Assume that

$$
\sum_{i=1}^{2 N} \frac{g_{i-1}+4 g_{i}+g_{i+1}}{3} h_{i}=\rho<1
$$

and mesh function $\psi\left(x_{i}\right)$ satisfy $\psi\left(x_{0}\right) \geq 0$ and $K^{N} \psi\left(x_{2 N}\right) \geq 0$. Then, $L_{1}^{N} \psi\left(x_{i}\right) \geq 0, \forall x_{i} \in \Omega_{1}^{2 N}$, $L_{2}^{N} \psi\left(x_{i}\right) \geq 0, \forall x_{i} \in \Omega_{2}^{2 N}$ and $D^{+}\left(\psi\left(x_{N}\right)\right)-D^{-}\left(\psi\left(x_{N}\right)\right) \leq 0$ imply that $\psi\left(x_{i}\right) \geq 0, \forall x_{i} \in \bar{\Omega}^{2 N}$.

Proof: Define 


$$
s\left(x_{i}\right)= \begin{cases}\frac{1}{8}+\frac{x_{i}}{2}, & x_{i} \in[0,1] \cap \bar{\Omega}^{2 N} \\ \frac{3}{8}+\frac{x_{i}}{4}, & x_{i} \in[1,2] \cap \bar{\Omega}^{2 N}\end{cases}
$$

Note that $s\left(x_{i}\right)>0, \forall x_{i} \in \bar{\Omega}^{2 N}, L s\left(x_{i}\right)>0, \forall x_{i} \in \Omega_{1}^{2 N} \cup \Omega_{2}^{2 N}, s(0)>0, K s\left(x_{2 N}\right)>0 \quad$, and $\left[s^{\prime}\right]\left(x_{N}\right)<0$.

Let $\mu=\max \left\{\frac{-\psi\left(x_{i}\right)}{s\left(x_{i}\right)}: x_{i} \in \bar{\Omega}^{2 N}\right\}$. Then, there exists $x_{k} \in \bar{\Omega}^{2 N}$ such that $\psi\left(x_{k}\right)+\mu s\left(x_{k}\right)=0$ and $\psi\left(x_{i}\right)+\mu s\left(x_{i}\right) \geq 0, \forall x_{i} \in \bar{\Omega}^{2 N}$. Therefore, the function $(\psi+\mu s)$ attains its minimum at $x=x_{k}$. Suppose the theorem does not hold true, then, $\mu>0$.

Case (i): $x_{k}=x_{0}, 0<(\psi+\mu s)\left(x_{0}\right)=0$, it is a contradiction.

Case (ii): $x_{k} \in \Omega_{1}^{2 N}, 0<L_{1}^{N}(\psi+\mu s)\left(x_{k}\right) \leq 0$, it is a contradiction.

Case (iii): $x_{k}=x_{N}, 0 \leq\left[D(\psi+\mu s)^{\prime}\right]\left(x_{N}\right)<0$, it is a contradiction.

Case (iv): $x_{k} \in \Omega_{2}^{2 N}, 0<L_{2}^{N}(\psi+\mu s)\left(x_{k}\right) \leq 0$, it is a contradiction.

Case (v): $x_{k}=x_{2 N}$

$$
\begin{aligned}
0 & <K^{N}(\psi+\mu s) x_{2 N} \\
& =(\psi+\mu s) x_{2 N^{-}} \sum_{i=1}^{2 N} \frac{g_{i-1}(\psi+\mu s) x_{i-1}+4 g_{i}(\psi+\mu s) x_{i}+g_{i+1}(\psi+\mu s) x_{i+1}}{3} h_{i} \leq 0
\end{aligned}
$$

It is a contradiction. Hence the proof of the theorem.

Lemma 6: Let $\psi\left(x_{i}\right)$ be any mesh function then for $0 \leq i \leq 2 N$,

$$
\left|\psi\left(x_{i}\right)\right| \leq C \max \left\{\left|\psi\left(x_{o}\right)\right|,\left|K^{N} \psi\left(x_{2 N}\right)\right|, \max _{i \in \Omega_{1}^{2 N} \cup \Omega_{2}^{2 N}}\left|L^{N} \psi\left(x_{i}\right)\right|\right\} .
$$

Proof: For the proof, refer to [16].

The following theorem shows the parameter uniform convergence of the scheme developed.

Theorem 1: Let $y\left(x_{i}\right)$ and $y_{i}$ be respectively the exact solution of (1)-(3) and numerical solutions of (17). Then, for sufficiently large $N$, the following parameter uniform error estimate holds:

$$
\sup _{0<\varepsilon \leq 1}\left\|y\left(x_{i}\right)-y_{i}\right\| \leq C N^{-2}
$$

Proof: Let us consider the local truncation error defined as follows:

$$
L^{h}\left(y\left(x_{i}\right)-y_{i}\right)=-\varepsilon \sigma(\rho)\left(\frac{d^{2}}{d x^{2}}-D^{+} D^{-}\right) y\left(x_{i}\right)+a\left(x_{i}\right)\left(\frac{d}{d x}-D^{0}\right) y\left(x_{i}\right),
$$

where $\varepsilon \sigma(\rho)=a(1) \frac{N^{-1}}{2} \operatorname{coth}\left(a(1) \frac{N^{-1}}{2 \varepsilon}\right)$ since $\rho=\frac{N^{-1}}{\varepsilon}$. In our assumption, $\varepsilon \leq h=N^{-1}$.

By considering $N$ is fixed and taking the limit for $\varepsilon \rightarrow 0$, we obtain the following:

$$
\lim _{\varepsilon \rightarrow 0} \varepsilon \sigma(\rho)=\lim _{\varepsilon \rightarrow 0} a(1) \frac{N^{-1}}{2} \operatorname{coth}\left(a(1) \frac{N^{-1}}{2 \varepsilon}\right)=C N^{-1} .
$$

From Taylor's series expansion, the bound for the difference becomes: 


$$
\left\{\begin{array}{l}
\left\|\left(\frac{d^{2}}{d x^{2}}-D^{+} D^{-}\right) y\left(x_{i}\right)\right\| \leq C N^{-3}\left\|\frac{d^{4}\left(y\left(x_{i}\right)\right)}{d x^{4}}\right\| \\
\left\|\left(\frac{d}{d x}-D^{0}\right) y\left(x_{i}\right)\right\| \leq C N^{-2}\left\|\frac{d^{3}\left(y\left(x_{i}\right)\right)}{d x^{3}}\right\|
\end{array}\right.
$$

where $\left\|\frac{d^{k}\left(y\left(x_{i}\right)\right)}{d x^{k}}\right\|=\sup _{x_{i} \in\left(x_{0}, x_{N}\right)}\left(\frac{d^{k} y\left(x_{i}\right)}{d x^{k}}\right), k=3,4$.

Now, using the bounds and the assumption $\varepsilon \leq N^{-1}$, (33) reduces to:

$$
\begin{aligned}
\left\|L^{h}\left(y\left(x_{i}\right)-y_{i}\right)\right\| & =\left\|-\varepsilon \sigma(\rho)\left(\frac{d^{2}}{d x^{2}}-D^{+} D^{-}\right) y\left(x_{i}\right)+a\left(x_{i}\right)\left(\frac{d}{d x}-D^{0}\right) y\left(x_{i}\right)\right\| \\
& \leq\left\|-\varepsilon \sigma(\rho)\left(\frac{d^{2}}{d x^{2}}-D^{+} D^{-}\right) y\left(x_{i}\right)\right\|+\left\|a\left(x_{i}\right)\left(\frac{d}{d x}-D^{0}\right) y\left(x_{i}\right)\right\| . \\
& \leq C N^{-3}\left\|\frac{d^{4}\left(y\left(x_{i}\right)\right)}{d x^{4}}\right\|+C N^{-2}\left\|\frac{d^{3}\left(y\left(x_{i}\right)\right)}{d x^{3}}\right\|
\end{aligned}
$$

Here, the target is to show the scheme convergence independent on the number of mesh points.

By using the bounds for the derivatives of the solution in Lemma 4, we obtain:

$$
\begin{aligned}
\left\|L^{h}\left(y\left(x_{i}\right)-y_{i}\right)\right\| \leq C N^{-3}\left\|\frac{d^{4}\left(y\left(x_{i}\right)\right)}{d x^{4}}\right\|+C N^{-2}\left\|\frac{d^{3}\left(y\left(x_{i}\right)\right)}{d x^{3}}\right\| \\
\leq C N^{-3}\left(1+\varepsilon^{-4} \exp \left(\frac{-\alpha\left(1-x_{j}\right)}{\varepsilon}\right)\right)+C N^{-2}\left(1+\varepsilon^{-3} \exp \left(\frac{-\alpha\left(1-x_{j}\right)}{\varepsilon}\right)\right) . \\
\leq C N^{-2}\left(1+\varepsilon^{-4} \exp \left(\frac{-\alpha\left(1-x_{j}\right)}{\varepsilon}\right)\right), \text { since } \varepsilon^{-4} \geq \varepsilon^{-3}
\end{aligned}
$$

Lemma 7: For a fixed mesh and for $\varepsilon \rightarrow 0$, it holds:

$$
\lim _{\varepsilon \rightarrow 0} \max _{1 \leq j \leq N-1} \frac{\exp \left(\frac{-\alpha\left(1-x_{j}\right)}{\varepsilon}\right)}{\varepsilon^{m}}=0, \quad m=1,2,3, \ldots
$$

Proof: Refer to [19].

By using Lemma 7 into (35), results to

$$
\left\|L^{h}\left(y\left(x_{i}\right)-y_{i}\right)\right\| \leq C N^{-2}
$$

Hence, by discrete maximum principle, we obtain:

$$
\left\|y\left(x_{i}\right)-y_{i}\right\| \leq C N^{-2} \text {. }
$$

Thus, result of (38) shows (32). Hence, the proof.

Remark: A similar analysis for convergence may be carried out for the finite difference scheme (24).

\section{Richardson Extrapolation}

This technique is acceleration technique which involves combination of two computed approximations of a solution. The combination goes out to be an improved approximation. From the local truncation term, we have: 


$$
\left|y\left(x_{i}\right)-y_{i}\right| \leq C(h)
$$

where $y\left(x_{i}\right)$ and $y_{i}$ are exact and approximate solutions respectively, and $C$ is constant free from mesh size $h$.

Let $\Omega^{4 N}$ be the mesh found by dividing each mesh interval in $\Omega^{2 N}$ and symbolize the calculation of the solution on $\Omega^{4 N}$ by $\bar{y}_{i}$. Consider (39) works for any $h \neq 0$, which implies:

$$
y\left(x_{i}\right)-y_{i} \leq C(h)+R^{2 N}, \quad x_{i} \in \Omega^{2 N}
$$

So that it works for any $\frac{h}{2} \neq 0$ yields:

$$
y\left(x_{i}\right)-\bar{y}_{i} \leq C\left(\frac{h}{2}\right)+R^{4 N}, \quad x_{i} \in \Omega^{4 N}
$$

where the remainders $R^{2 N}$ and $R^{4 N}$ are $O\left(h^{2}\right)$. Combination of inequalities in (40) and (41) leads to $y\left(x_{i}\right)-\left(2 \bar{y}_{i}-y_{i}\right) \approx O\left(h^{2}\right)$ which proposes that

$$
\left(y_{i}\right)^{e x t}=2 \bar{y}_{i}-y_{i}
$$

is also a rough calculation of $y\left(x_{i}\right)$. By means of this approximation to estimate the truncation error, we obtain:

$$
\left|y\left(x_{i}\right)-\left(y_{i}\right)^{e x t}\right| \leq C\left(h^{2}\right)
$$

where $C$ is free of mesh size $h$. Thus, using Richardson extrapolation first order convergent method is accelerated into second order convergent as provided in (43). Thus, we can say that the proposed method is second order convergent.

\section{Numerical examples and results}

In this section, two examples are considered to illustrate the applicability of the numerical method discussed above. The exact solutions of these test problems are not known. Therefore, double mesh principle is used to estimate the errors and compute the numerical rate of convergence to the computed solution. The double mesh formula to determine maximum absolute error is defined as follows:

$$
E_{\varepsilon}^{h}=\max _{0 \leq i \leq 2 N}\left|Y_{i}^{N}-Y_{2 i}^{2 N}\right|
$$

where $Y_{i}^{N}$ and $Y_{2 i}^{2 N}$ are the $i^{t h}$ components of the numerical solutions for $N$ and $2 N$, respectively. We compute the uniform error and the rate of convergence using the formula:

$$
E^{h}=\max _{\varepsilon} E_{\varepsilon}^{h} \text { and } R^{h}=\log _{2}\left(\frac{E^{N}}{E^{2 N}}\right)
$$

The numerical results are presented for the values of the perturbation parameter $\varepsilon \in\left\{10^{-4}, 10^{-8}, \ldots, 10^{-20}\right\}$.

Example 1: 
Table 1 Maximum absolute errors for Example 1 at number of mesh points $2 \mathrm{~N}$

\begin{tabular}{llllll}
\hline$\varepsilon$ & $N=32$ & $N=64$ & $N=128$ & $N=256$ & $N=512$ \\
\hline $10^{-4}$ & $6.8161 \mathrm{e}-06$ & $1.7125 \mathrm{e}-06$ & $4.2918 \mathrm{e}-07$ & $1.0743 \mathrm{e}-07$ & $2.6988 \mathrm{e}-08$ \\
& 1.9928 & 1.9964 & 1.9982 & 1.9930 & \\
$10^{-8}$ & $6.8161 \mathrm{e}-06$ & $1.7125 \mathrm{e}-06$ & $4.2918 \mathrm{e}-07$ & $1.0743 \mathrm{e}-07$ & $2.6988 \mathrm{e}-08$ \\
& 1.9928 & 1.9964 & 1.9982 & 1.9930 & \\
$10^{-12}$ & $6.8161 \mathrm{e}-06$ & $1.7125 \mathrm{e}-06$ & $4.2918 \mathrm{e}-07$ & $1.0743 \mathrm{e}-07$ & $2.6988 \mathrm{e}-08$ \\
& 1.9928 & 1.9964 & 1.9982 & 1.9930 & \\
$10^{-16}$ & $6.8161 \mathrm{e}-06$ & $1.7125 \mathrm{e}-06$ & $4.2918 \mathrm{e}-07$ & $1.0743 \mathrm{e}-07$ & $2.6988 \mathrm{e}-08$ \\
& 1.9928 & 1.9964 & 1.9982 & 1.9930 & \\
$10^{-20}$ & $6.8161 \mathrm{e}-06$ & $1.7125 \mathrm{e}-06$ & $4.2918 \mathrm{e}-07$ & $1.0743 \mathrm{e}-07$ & $2.6988 \mathrm{e}-08$ \\
& 1.9928 & 1.9964 & 1.9982 & 1.9930 & \\
\hline
\end{tabular}

Table 2 Comparison of maximum absolute errors for Example 1 at number of mesh points $2 \mathrm{~N}$

\begin{tabular}{|c|c|c|c|c|c|}
\hline$\varepsilon \downarrow N \rightarrow$ & 32 & 64 & 128 & 256 & 512 \\
\hline \multicolumn{6}{|c|}{ Present method } \\
\hline $2^{-10}$ & $6.8161 \mathrm{e}-06$ & $1.7253 e-06$ & $6.3777 \mathrm{e}-06$ & $5.5050 \mathrm{e}-05$ & $6.1658 \mathrm{e}-05$ \\
\hline $2^{-11}$ & $6.8161 \mathrm{e}-06$ & $1.7125 \mathrm{e}-06$ & $4.3561 \mathrm{e}-07$ & $1.1065 \mathrm{e}-07$ & $1.6001 \mathrm{e}-06$ \\
\hline $2^{-12}$ & $6.8161 \mathrm{e}-06$ & $1.7125 \mathrm{e}-06$ & $4.2918 \mathrm{e}-07$ & $1.0743 e-07$ & $2.6874 \mathrm{e}-08$ \\
\hline $2^{-13}$ & $6.8161 \mathrm{e}-06$ & $1.7125 \mathrm{e}-06$ & $4.2918 \mathrm{e}-07$ & $1.0743 \mathrm{e}-07$ & $2.6874 \mathrm{e}-08$ \\
\hline $2^{-14}$ & $6.8161 \mathrm{e}-06$ & $1.7125 \mathrm{e}-06$ & $4.2918 \mathrm{e}-07$ & $1.0743 \mathrm{e}-07$ & $2.6874 \mathrm{e}-08$ \\
\hline $2^{-15}$ & $6.8161 \mathrm{e}-06$ & $1.7125 \mathrm{e}-06$ & $4.2918 \mathrm{e}-07$ & $1.0743 \mathrm{e}-07$ & $2.6874 \mathrm{e}-08$ \\
\hline $2^{-16}$ & $6.8161 \mathrm{e}-06$ & $1.7125 \mathrm{e}-06$ & $4.2918 \mathrm{e}-07$ & $1.0743 \mathrm{e}-07$ & $2.6874 \mathrm{e}-08$ \\
\hline $2^{-17}$ & 6.8161e-06 & $1.7125 \mathrm{e}-06$ & $4.2918 \mathrm{e}-07$ & $1.0743 e-07$ & $2.6874 \mathrm{e}-08$ \\
\hline $2^{-18}$ & $6.8161 \mathrm{e}-06$ & $1.7125 \mathrm{e}-06$ & $4.2918 \mathrm{e}-07$ & $1.0743 \mathrm{e}-07$ & $2.6874 \mathrm{e}-08$ \\
\hline $2^{-19}$ & $6.8161 \mathrm{e}-06$ & $1.7125 \mathrm{e}-06$ & $4.2918 \mathrm{e}-07$ & $1.0743 \mathrm{e}-07$ & $2.6874 \mathrm{e}-08$ \\
\hline $2^{-20}$ & $6.8161 \mathrm{e}-06$ & $1.7125 \mathrm{e}-06$ & $4.2918 \mathrm{e}-07$ & $1.0743 \mathrm{e}-07$ & $2.6874 \mathrm{e}-08$ \\
\hline$E_{N}$ & $6.8161 \mathrm{e}-06$ & $1.7125 \mathrm{e}-06$ & $4.2918 \mathrm{e}-07$ & $1.0743 \mathrm{e}-07$ & $2.6874 \mathrm{e}-08$ \\
\hline$R_{N}$ & 1.9821 & 1.9964 & 1.9982 & 1.9991 & \\
\hline \multicolumn{6}{|c|}{ Result in [16] } \\
\hline $2^{-10}$ & $5.0010 \mathrm{e}-03$ & $2.3755 e-03$ & $1.1275 e-03$ & $5.3851 \mathrm{e}-04$ & $2.6147 e-04$ \\
\hline $2^{-11}$ & $5.3378 \mathrm{e}-03$ & $2.5881 \mathrm{e}-03$ & $1.2538 \mathrm{e}-03$ & $6.0996 \mathrm{e}-04$ & $2.9986 \mathrm{e}-04$ \\
\hline $2^{-12}$ & $5.5738 \mathrm{e}-03$ & $2.7367 e-03$ & $1.3418 \mathrm{e}-03$ & $6.5956 \mathrm{e}-04$ & $3.2641 \mathrm{e}-04$ \\
\hline $2^{-13}$ & 5.7397e-03 & $2.8409 \mathrm{e}-03$ & $1.4034 \mathrm{e}-03$ & $6.9419 e-04$ & $3.4487 \mathrm{e}-04$ \\
\hline $2^{-14}$ & $5.8565 \mathrm{e}-03$ & $2.9141 \mathrm{e}-03$ & $1.4466 \mathrm{e}-03$ & $7.1844 \mathrm{e}-04$ & $3.5778 \mathrm{e}-04$ \\
\hline $2^{-15}$ & $5.9389 \mathrm{e}-03$ & $2.9657 \mathrm{e}-03$ & $1.4770 \mathrm{e}-03$ & $7.3548 \mathrm{e}-04$ & $3.6683 \mathrm{e}-04$ \\
\hline $2^{-16}$ & $5.9970 \mathrm{e}-03$ & $3.0021 \mathrm{e}-03$ & $1.4985 \mathrm{e}-03$ & 7.4747e-04 & $3.7319 \mathrm{e}-04$ \\
\hline $2^{-17}$ & $6.0380 \mathrm{e}-03$ & $3.0278 \mathrm{e}-03$ & $1.5136 \mathrm{e}-03$ & 7.5592e-04 & $3.7767 e-04$ \\
\hline $2^{-18}$ & $6.0670 \mathrm{e}-03$ & $3.0459 \mathrm{e}-03$ & $1.5242 \mathrm{e}-03$ & $7.6189 \mathrm{e}-04$ & $3.8083 \mathrm{e}-04$ \\
\hline $2^{-19}$ & $6.0875 e-03$ & $3.0587 \mathrm{e}-03$ & $1.5318 \mathrm{e}-03$ & $7.6610 \mathrm{e}-04$ & $3.8306 \mathrm{e}-04$ \\
\hline $2^{-20}$ & $6.1020 \mathrm{e}-03$ & $3.0678 \mathrm{e}-03$ & $1.5371 \mathrm{e}-03$ & 7.6907e-04 & $3.8464 \mathrm{e}-04$ \\
\hline$E_{N}$ & $6.1020 \mathrm{e}-03$ & $3.0678 \mathrm{e}-03$ & $1.5371 \mathrm{e}-03$ & $7.6907 e-04$ & $3.8464 \mathrm{e}-04$ \\
\hline$R_{N}$ & 0.99207 & 0.99697 & 0.99904 & 0.99960 & \\
\hline
\end{tabular}


Table 3 Maximum absolute errors for Example 2 at number of mesh points $2 \mathrm{~N}$

\begin{tabular}{llllll}
\hline$\varepsilon$ & $N=32$ & $N=64$ & $N=128$ & $N=256$ & $N=512$ \\
\hline $10^{-4}$ & $3.5556 \mathrm{e}-05$ & $8.7577 \mathrm{e}-06$ & $2.1733 \mathrm{e}-06$ & $5.4134 \mathrm{e}-07$ & $1.3509 \mathrm{e}-07$ \\
& 2.0215 & 2.0107 & 2.0053 & 2.0026 & \\
$10^{-8}$ & $3.5556 \mathrm{e}-05$ & $8.7577 \mathrm{e}-06$ & $2.1733 \mathrm{e}-06$ & $5.4134 \mathrm{e}-07$ & $1.3509 \mathrm{e}-07$ \\
& 2.0215 & 2.0107 & 2.0053 & 2.0026 & \\
$10^{-12}$ & $3.5556 \mathrm{e}-05$ & $8.7577 \mathrm{e}-06$ & $2.1733 \mathrm{e}-06$ & $5.4134 \mathrm{e}-07$ & $1.3509 \mathrm{e}-07$ \\
& 2.0215 & 2.0107 & 2.0053 & 2.0026 & \\
$10^{-16}$ & $3.5556 \mathrm{e}-05$ & $8.7577 \mathrm{e}-06$ & $2.1733 \mathrm{e}-06$ & $5.4134 \mathrm{e}-07$ & $1.3509 \mathrm{e}-07$ \\
& 2.0215 & 2.0107 & 2.0053 & 2.0026 & \\
$10^{-20}$ & $3.5556 \mathrm{e}-05$ & $8.7577 \mathrm{e}-06$ & $2.1733 \mathrm{e}-06$ & $5.4134 \mathrm{e}-07$ & $1.3509 \mathrm{e}-07$ \\
& 2.0215 & 2.0107 & 2.0053 & 2.0026 & \\
\hline
\end{tabular}

Table 4 Comparison of maximum absolute errors for Example 2 at number of mesh points $2 \mathrm{~N}$

\begin{tabular}{|c|c|c|c|c|c|}
\hline$\varepsilon \downarrow N \rightarrow$ & 32 & 64 & 128 & 256 & 512 \\
\hline \multicolumn{6}{|c|}{ Present method } \\
\hline $2^{-10}$ & $3.5556 \mathrm{e}-05$ & $8.7577 \mathrm{e}-06$ & $2.2227 \mathrm{e}-06$ & $4.1781 \mathrm{e}-06$ & $1.9844 \mathrm{e}-05$ \\
\hline $2^{-11}$ & $3.5556 \mathrm{e}-05$ & $8.7577 e-06$ & $2.1733 \mathrm{e}-06$ & $5.4134 \mathrm{e}-07$ & $1.4748 \mathrm{e}-07$ \\
\hline $2^{-12}$ & $3.5556 \mathrm{e}-05$ & 8.7577e-06 & $2.1733 e-06$ & $5.4134 \mathrm{e}-07$ & $1.3509 e-07$ \\
\hline $2^{-13}$ & $3.5556 \mathrm{e}-05$ & $8.7577 \mathrm{e}-06$ & $2.1733 \mathrm{e}-06$ & $5.4134 \mathrm{e}-07$ & $1.3509 \mathrm{e}-07$ \\
\hline $2^{-14}$ & $3.5556 \mathrm{e}-05$ & $8.7577 e-06$ & $2.1733 e-06$ & $5.4134 \mathrm{e}-07$ & $1.3509 \mathrm{e}-07$ \\
\hline $2^{-15}$ & $3.5556 \mathrm{e}-05$ & $8.7577 \mathrm{e}-06$ & $2.1733 e-06$ & $5.4134 \mathrm{e}-07$ & $1.3509 \mathrm{e}-07$ \\
\hline $2^{-16}$ & $3.5556 \mathrm{e}-05$ & $8.7577 e-06$ & $2.2227 \mathrm{e}-06$ & $5.4134 \mathrm{e}-07$ & $1.3509 \mathrm{e}-07$ \\
\hline $2^{-17}$ & $3.5556 \mathrm{e}-05$ & $8.7577 \mathrm{e}-06$ & $2.1733 e-06$ & $5.4134 \mathrm{e}-07$ & $1.3509 \mathrm{e}-07$ \\
\hline $2^{-18}$ & $3.5556 \mathrm{e}-05$ & $8.7577 \mathrm{e}-06$ & $2.1733 \mathrm{e}-06$ & $5.4134 \mathrm{e}-07$ & $1.3509 \mathrm{e}-07$ \\
\hline $2^{-19}$ & $3.5556 \mathrm{e}-05$ & $8.7577 \mathrm{e}-06$ & $2.1733 e-06$ & $5.4134 \mathrm{e}-07$ & $1.3509 e-07$ \\
\hline $2^{-20}$ & $3.5556 \mathrm{e}-05$ & $8.7577 e-06$ & $2.1733 e-06$ & $5.4134 \mathrm{e}-07$ & $1.3509 \mathrm{e}-07$ \\
\hline$E_{N}$ & $3.5556 \mathrm{e}-05$ & $8.7577 \mathrm{e}-06$ & $2.1733 e-06$ & $5.4134 \mathrm{e}-07$ & $1.3509 \mathrm{e}-07$ \\
\hline$R_{N}$ & 2.0215 & 2.0107 & 2.0053 & 2.0026 & \\
\hline \multicolumn{6}{|c|}{ Result in [16] } \\
\hline $2^{-10}$ & $1.5023 e-03$ & $8.2944 \mathrm{e}-04$ & $4.2515 \mathrm{e}-04$ & $2.1093 e-04$ & $1.0303 e-04$ \\
\hline $2^{-11}$ & $1.5379 \mathrm{e}-03$ & $8.6376 \mathrm{e}-04$ & $4.4918 \mathrm{e}-04$ & $2.2591 \mathrm{e}-04$ & $1.1186 \mathrm{e}-04$ \\
\hline $2^{-12}$ & $1.5610 \mathrm{e}-03$ & $8.8702 \mathrm{e}-04$ & $4.6570 \mathrm{e}-04$ & $2.3629 \mathrm{e}-04$ & $1.1800 \mathrm{e}-04$ \\
\hline $2^{-13}$ & $1.5763 e-03$ & $9.0297 \mathrm{e}-04$ & $4.7715 e-04$ & $2.4351 e-04$ & $1.2228 \mathrm{e}-04$ \\
\hline $2^{-14}$ & $1.5865 \mathrm{e}-03$ & $9.1398 \mathrm{e}-04$ & $4.8511 \mathrm{e}-04$ & $2.4855 \mathrm{e}-04$ & $1.2528 \mathrm{e}-04$ \\
\hline $2^{-15}$ & $1.5935 \mathrm{e}-03$ & $9.2163 e-04$ & 4.9067e-04 & $2.5209 \mathrm{e}-04$ & $1.2738 \mathrm{e}-04$ \\
\hline $2^{-16}$ & $1.5983 e-03$ & $9.2698 \mathrm{e}-04$ & $4.9458 \mathrm{e}-04$ & $2.5457 \mathrm{e}-04$ & $1.2885 \mathrm{e}-04$ \\
\hline $2^{-17}$ & $1.6016 \mathrm{e}-03$ & $9.3072 \mathrm{e}-04$ & $4.9732 \mathrm{e}-04$ & $2.5631 \mathrm{e}-04$ & $1.2989 \mathrm{e}-04$ \\
\hline $2^{-18}$ & $1.6039 \mathrm{e}-03$ & $9.3336 \mathrm{e}-04$ & $4.9925 \mathrm{e}-04$ & $2.5754 \mathrm{e}-04$ & $1.3063 e-04$ \\
\hline $2^{-19}$ & $1.6055 \mathrm{e}-03$ & $9.3521 \mathrm{e}-04$ & $5.0061 e-04$ & $2.5841 \mathrm{e}-04$ & $1.3114 \mathrm{e}-04$ \\
\hline $2^{-20}$ & $1.6067 \mathrm{e}-03$ & $9.3652 \mathrm{e}-04$ & $5.0157 \mathrm{e}-04$ & $2.5902 \mathrm{e}-04$ & $1.3151 \mathrm{e}-04$ \\
\hline$E_{N}$ & $1.6067 \mathrm{e}-03$ & $9.3652 \mathrm{e}-04$ & $5.0157 e-04$ & $2.5902 \mathrm{e}-04$ & $1.3151 \mathrm{e}-04$ \\
\hline$R_{N}$ & 1.0602 & 1.0317 & 1.0162 & 1.0082 & \\
\hline
\end{tabular}




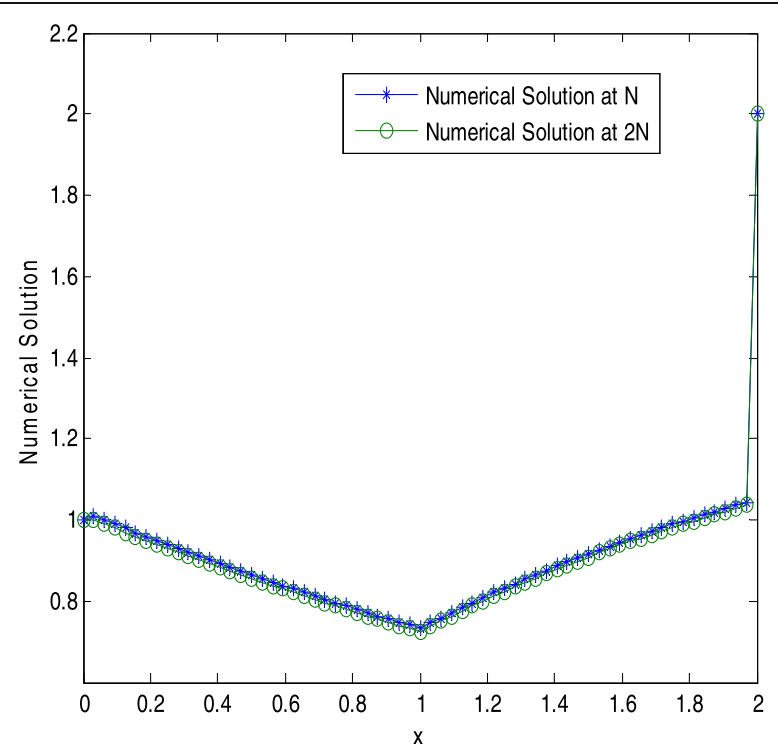

Fig. 1 The behavior of the numerical solution for Example 1 at $\varepsilon=10^{-12}$ and $N=32$

$$
\begin{aligned}
& -\varepsilon y^{\prime \prime}(x)+3 y^{\prime}(x)+y(x)-y(x-1)=1, \quad x \in(0,1) \cup(1,2) \\
& y(x)=1, \quad x \in[-1,0] \\
& y(2)-\varepsilon \int_{0}^{2} \frac{x}{3} y(x) d x=2
\end{aligned}
$$

Example 2:

$$
\begin{aligned}
& -\varepsilon y^{\prime \prime}(x)+5 y^{\prime}(x)+(x+1) y(x)-y(x-1)=x^{2}, \quad x \in(0,1) \cup(1,2) \\
& y(x)=1, \quad x \in[-1,0] \\
& y(2)-\varepsilon \int_{0}^{2} \frac{x}{3} y(x) d x=2
\end{aligned}
$$

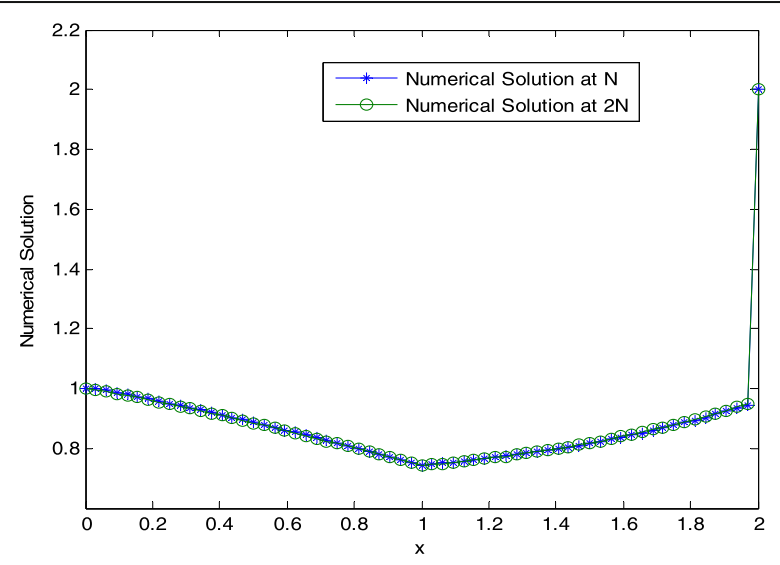

Fig. 2 The behavior of the numerical solution for Example 2 at $\varepsilon=10^{-12}$ and $N=32$ 


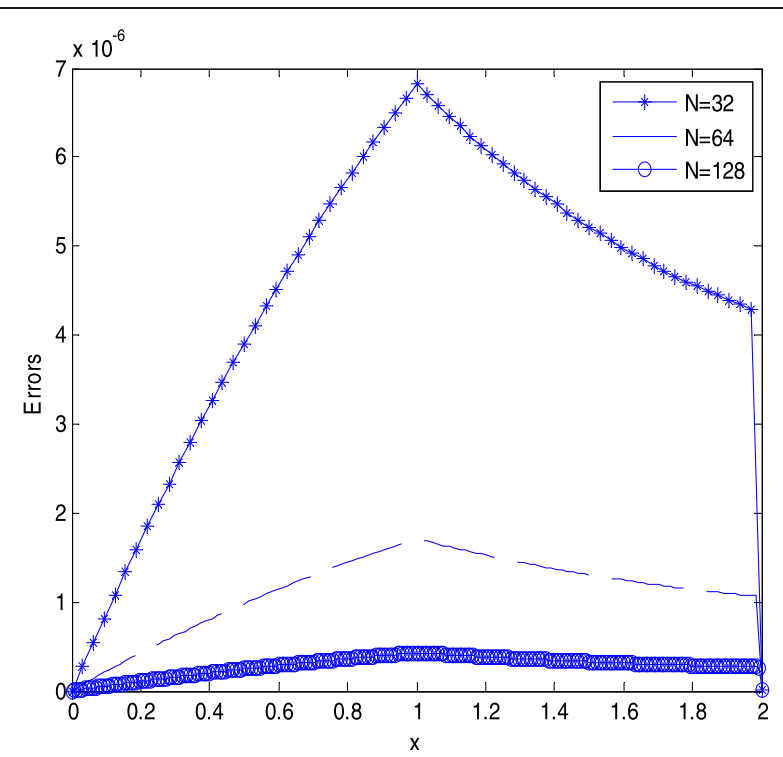

Fig. 3 Point wise absolute error of Example 1 at $\varepsilon=10^{-12}$ with different mesh points $N$

\section{Discussion and conclusion}

This study introduces accelerated fitted operator numerical method for solving singularly perturbed delay differential equations with integral boundary condition. The behavior of the continuous solution of the problem is studied and shown that it satisfies the continuous stability estimate, and the derivatives of the solution are also bounded. The numerical scheme is developed on uniform mesh using fitted operator finite difference method in the given differential equation. The integral boundary condition is treated by using Simpson's rule. The stability of the developed numerical method is established, and its uniform convergence is proved. To validate the applicability of the method, two model problems are considered for numerical experimentation for different values of the perturbation parameter and mesh points. The numerical results are tabulated in terms of maximum absolute errors, numerical rate of convergence, and uniform errors (see Tables 1, 2, 3 and 4). Further, Figs. 1 and 2 show that for small values of $\varepsilon$, the solution of the problem under consideration exhibits strong boundary

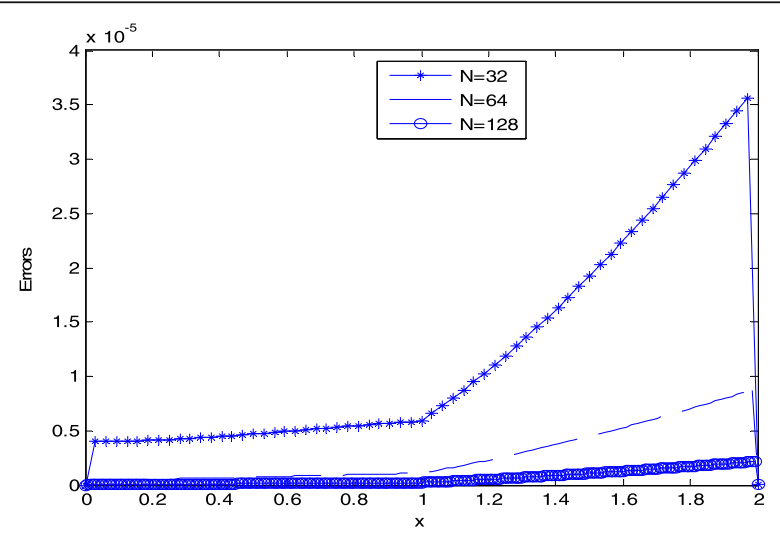

Fig. 4 Point wise absolute error of Example 2 at $\varepsilon=10^{-12}$ with different mesh points $\mathrm{N}$ 


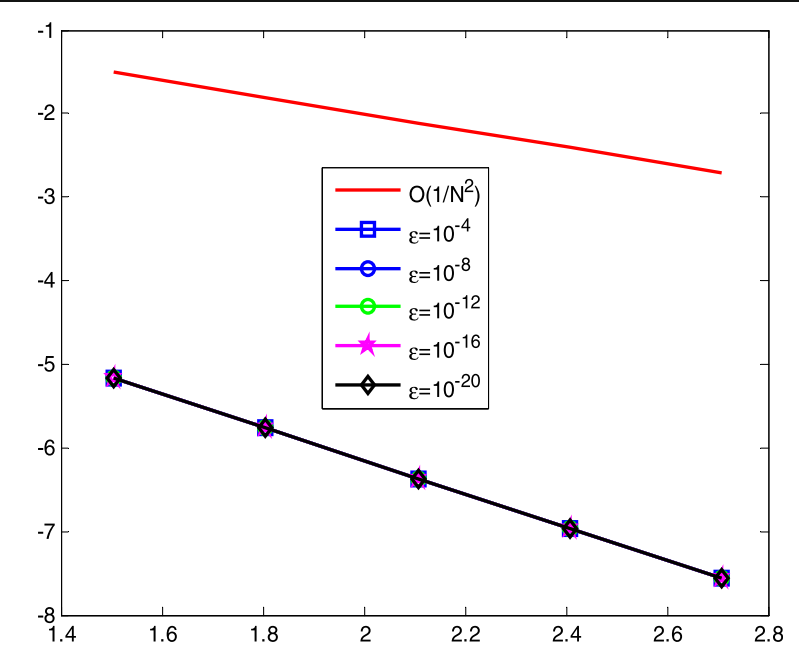

Fig. 5 -uniform convergence with fitted operator in log-log scale for Example 1

layer at $x=2$, and interior layer at $x=1$. Figures 3 and 4 show that as the mesh size decrease or as the number of mesh point increase, the absolute error decreases. The log$\log$ scale plot in Figs. 5 and 6 depicted the $\varepsilon$-uniformly convergence of the method for $h \geq \varepsilon$ where the classical numerical methods fail to converge. The method is shown to be $\varepsilon$-uniformly convergent with order of convergence $O\left(h^{2}\right)$. The performance of the proposed scheme is investigated by comparing with prior study (Tables 2 and 4). The proposed method is stable, more accurate, and convergent independent of the values of the perturbation parameter and the mesh size. The authors suggested that one can extend the work or solve the problem under consideration by applying higher order fitted operator numerical methods or Bakhavlov-type fitted mesh numerical method to obtain more accurate numerical results.

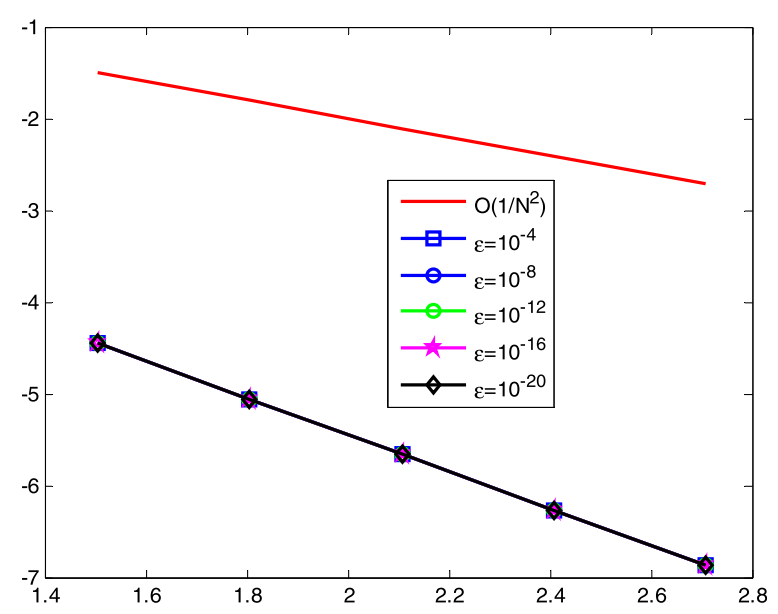

Fig. $6 \varepsilon$-uniform convergence with fitted operator in log-log scale for Example 2 


\section{Acknowledgements}

The authors wish to express their thanks to the authors of literatures for the provision of initial idea for this work. We also thank Jimma University for the necessary support.

\section{Authors' contributions}

HGD proposed the main idea of this paper. HGD and GFD prepared the manuscript and performed all the steps of the proofs in this research. Both authors contributed equally and significantly in writing this paper. Both authors read and approved the final manuscript.

\section{Funding}

Not applicable.

\section{Availability of data and materials}

All data generated or analyzed during this study are included.

\section{Competing interests}

The authors declare that they have no competing interests.

Received: 11 December 2019 Accepted: 2 March 2020

Published online: 30 March 2020

\section{References}

1. Longtin, A., Milton, J.: Complex oscillations in the human pupil light reflex with mixed and delayed feedback. Math. Biosci. 90, 183-199 (1988)

2. Glizer, V.Y: Asymptotic analysis and solution of a finite-horizon $\mathrm{H}^{\alpha}$ control problem for singularly perturbed linear systems with small state delay. J. Optim. Theory Appl. 117, 295-325 (2003)

3. Culshaw, R.V., Ruan, S.: A delay differential equation model of HIV infection of C D4+ T-cells. Math. Biosci. 165, 27-39 (2000)

4. Els'gol'ts, E.L.: Qualitative methods in mathematical analysis in: translations of mathematical monographs, vol. 12. American Mathematical Society, Providence (1964)

5. Amiraliyev, G.M., Amiraliyev, I.G., Kudu, M.: A numerical treatment for singularly perturbed differential equations with integral boundary condition. Appl. Math. Comput. 185(1), 574-582 (2007)

6. Cen, Z., Cai, X:: A second order upwind difference scheme for a singularly perturbed problem with integral boundary condition in netural network, pp. 175-181. Springer, Berlin (2007)

7. Kudu, M., Amiraliyev, G.: Finite difference method for a singularly perturbed differential equations with integra boundary condition. Int. J. Math. Comput. 26(3), 71-79 (2015)

8. Boucherif, A.: Second order boundary value problems with integral boundary condition. Nonlinear Anal. 70(1), 364-371 (2009)

9. Feng, M., Ji, D., Weigao, G.: Positive solutions for a class of boundary value problem with integral boundary conditions in banach spaces. J. Comput. Appl. Math. 222(2), 351-363 (2008)

10. Li, H., Sun, F.: Existence of solutions for integral boundary value problems of second order ordinary differential equations, Bound. Value Probl. 2012(1), 147 (2012)

11. Amiraliyev, G.M., Cimen, E.: Numerical method for a singularly perturbed convection-diffusion problem with delay. Appl. Math. Comput. 216(8), 2351-2359 (2010)

12. Mahendran, R., Subburayan, V.: Fitted finite difference method for third order singularly perturbed delay differential equations of convection diffusion type. Int. J. Comput. Methods. 16(5), 1840007 (2019)

13. Nicaise, S., Xenophontos, C.: Robust approximation of singularly perturbed delay differential equations by the hp finite element method. Comput. Methods Appl. Math. 13(1), 21-37 (2013)

14. Tang, Z.Q., Geng, F.Z.: Fitted reproducing kernel method for singularly perturbed delay initial value problems. Appl. Math. Comput. 284, 169-174 (2016)

15. Zarin, H.: On discontinuous Galerkin finite element method for singularly perturbed delay differential equations. Appl. Math. Lett. 38, 27-32 (2014)

16. Sekar, E., Tamilselvan, A.: Singularly perturbed delay differential equations of convection-diffusion type with integral boundary condition. J. Appl. Math. Comput. 59(1-2), 701-722 (2019). https://doi.org/10.1007/s12190-018-1198-4

17. Clavero, C., Gracia, J.L., Jorge, J.C.: High-order numerical methods for one dimensional parabolic singularly perturbed problems with regular layers. Numer. Methods Partial differential equations. 21(1), 149-169 (2005)

18. R.E. OMalley, Singular perturbation methods for ordinary differential equations. Springer-Verlag, New York, 89(1991).

19. Woldaregay, M.M., Duressa, G.F.: Parameter uniform numerical method for singularly perturbed differential difference equations. J. Nigerian Math. Soc. 38(2), 223-245 (2019)

\section{Publisher's Note}

Springer Nature remains neutral with regard to jurisdictional claims in published maps and institutional affiliations. 